Aims To review whether ultrasounds are being performed for Hospice at Home $(\mathrm{H} @ \mathrm{H})$ patients and what impact this has. Method A retrospective service evaluation of the use of the ultrasound in $\mathrm{H} @ \mathrm{H}$ patients over a one-year period.

Results 10 ultrasounds were performed in $\mathrm{H} @ \mathrm{H}$ patients over one year, seven were in the patient's home and three were in hospice outpatient appointments. Nine ultrasounds were performed to assess for ascites and one to assess for urinary retention.

Of the nine ultrasounds performed to assess for ascites, three demonstrated large volume of ascites that was amenable to drainage. Of these three patients, one had a drain inserted on the hospice inpatient unit and two were referred to hospital for drainage. The other six patients were found to have small volume or no ascites. The patient who had an ultrasound to assess for urinary retention was found not to have a distended bladder.

Conclusion The use of ultrasound in $\mathrm{H} @ \mathrm{H}$ patients does influence patient care and supports clinical decision making. The value of hospices performing ultrasounds comes from the ability to avoid unnecessary visits to hospital for ultrasounds, as well as avoiding unnecessary admissions to the hospice inpatient unit or hospital for assessment or drainage of ascites. Rapidly establishing whether a patient has ascites and whether it is amenable to drainage in the community, reduces delays and distress for the patient, as well as reducing the burden on the wider health service.

\section{P-169 WEIGHING PATIENTS IN A HOSPICE SETTING}

${ }^{1} J a n$ Codling, 'Jennie Pickard, 'Alison Phippen, 'Samantha Kay, ${ }^{2}$ Niall Byrne, ${ }^{1}$ Becki Singh, ${ }^{1}$ David Waterman. 'St Ann's Hospice, Heald Green, UK; ${ }^{2}$ Manchester University, Manchester, UK

\subsection{6/bmjspcare-2017-hospice.194}

Background Standard practice at our hospice did not encourage routine weighing of patients on admission, which potentially limited ability to meet best practice standards for medicine management and nutritional assessment.

Aim This project measured if patients were weighed at or soon after admission. Many were prescribed medication where dose was dependent on weight. The opinions of staff and patients towards routine weighing was also investigated.

Methods An audit of 40 patients measured if patients were weighed on admission or a reason for not doing so recorded and whether weight dependent doses were in line with the British National Formulary or other specialist advice.

A staff questionnaire gained the opinions of 79 clinical staff towards weighing patients, their understanding of the reason for weighing, and rationale for their opinions. A patient questionnaire gained the opinions of 38 patients on being weighed and their understanding of the reason for being weighed.

Results 97\% patients did not find being weighed distressing. However, 51\% staff members were opposed to routine weighing. $13 \%$ of patients had a weight recorded. $13 \%$ were prescribed low molecular weight heparin, $80 \%$ of these patients were weighed and only $60 \%$ were on the correct dose.

Conclusions Routine weighing has been introduced for all patients or a recorded reason for not doing so. Clinical staff now receive training that demonstrates the inaccuracy of estimating body weight. An alert sticker is now attached to the medicine chart, for patients prescribed weight dependant medication and a prompt on the shelves where the medication is stored acts as a reminder to check body weight.

There is a plan for regular audits of the weighing of patients to maintain the profile of the importance of weighing and these results will be fed back real time to the clinical teams.

\section{P-170 DETECTING SUICIDAL THOUGHTS IN PALLIATIVE CARE: WHEN DO PATIENTS VOICE THESE THOUGHTS?}

${ }^{1}$ Chloe Choy, ${ }^{2}$ Kathy Burn, ${ }^{2}$ Emma McLachlan, ${ }^{2}$ Emma Hall. ${ }^{1}$ King's College London, London, UK; ${ }^{2}$ St Christopher's Hospice, London, UK

\subsection{6/bmjspcare-2017-hospice. 195}

Background Early identification of suicidal ideation would allow more opportunities for intervention and may ultimately reduce risk of suicide. Knowing when patients are likely to voice suicidal thoughts is important, as it may increase confidence of healthcare professionals to have earlier conversations with their patients regarding suicide risk.

Aim To establish the time period between first assessment and recorded suicidal ideation in a patient population.

Methods 385 patients under the care of inpatient and community palliative care teams at St Christopher's Hospice, Sydenham, were identified to have keyword 'suicide' in their electronic patient records (EPR) during the period of April 2015 to March 2016. In these individuals, EPR was reviewed against inclusion criteria and 124 patients were identified to have documented suicidal ideation. Time from first assessment to detection of suicidal thoughts was calculated.

Results 61\% of patients with expressed suicidal thoughts $(n=76)$ were male, and mean age of those who voiced suicidal thoughts was $67.4 \pm 13.6$ years (mean \pm SD). $69 \%$ $(n=86)$ had a diagnosis of malignancy, and 62\% $(n=77)$ had a previous psychiatric history. $15 \%(n=19)$ of all patients we identified with suicidal thoughts voiced their suicidal ideation at their first assessment. A further 10\% $(n=12)$ voiced these thoughts within the first week of contact, and in $45 \% \quad(n=55)$ suicidal thoughts had been detected within the first month.

Conclusions A large proportion of patients expressed their suicidal ideation early in their contact with the hospice-centred palliative care team. As previously established, asking patients about thoughts of suicide does not increase risk of suicide or self-harm (Gould et al., 2005; Eynan et al., 2014). This data provides further encouragement to explore suicidal thoughts in palliative care patients from first contact.

\section{P-171 COMFORT ROUNDS: TASK-ORIENTATED NURSING OR EFFECTIVE CARE?}

Jo Carby, Rose Davis, Rebekah Ashley, Debbie Bewley, Nathalie Ward, Yvonne Tague. Wigan and Leigh Hospice, Greater Manchester, UK

\subsection{6/bmjspcare-2017-hospice. 196}

Background Active nursing rounds, also known as intentional, care or comfort rounds were first developed in the USA (Struder Group, 2007) and later introduced into UK hospitals in 2012 alongside other measures to improve the quality of nursing care (www.harmfreecare.org). They have been associated with reductions in pressure ulcers, falls and increased 
patient and staff satisfaction (Cornwell, 2012). A pressure ulcer audit (Hospice UK, 2016) on a hospice inpatient unit (IPU) highlighted the need to improve nurses' skills in the prevention, management and documentation of skin integrity.

Aim To design a comfort round tool specifically for use within the hospice IPU, implement comfort rounds and evaluate the impact on patients and nurses.

Method Nurses on the IPU were consulted regarding the overall concept, naming and design of the tool. Training was provided and following implementation, feedback sought from IPU nurses and patients regarding the impact of comfort rounds on patient care and experience.

Results Nurses' feedback: Healthcare assistants experienced job satisfaction with their involvement in continuity of patient care and evidencing care they provided. They felt areas for improvement included managing time pressures, patient choice, document design, training and team working. Patient feedback: 14 responses, all of whom were positive about the comfort rounds. They felt areas for improvement included communication about the rounds, patient choice and ensuring these discussions are communicated across the team.

Conclusion Comfort rounds have enabled our patients to feel less isolated and provided reassurances to family members. They support the consistent nursing care of our patients, including improving the involvement of the healthcare assistant workforce, optimising hydration and the prevention of pressure ulcers and falls. Initial assessment of patients admitted to the IPU needs to include discussion about the comfort rounds and creating individualised plans of care including the patient's needs and preferences relating to comfort rounds.

\section{P-172 PSYCHOSOCIAL EXPERIENCE OF WEIGHT LOSS IN CANCER CACHEXIA. WHAT CAN HEALTH CARE PROFESSIONALS LEARN?}

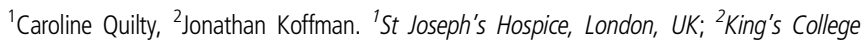
London, Cicely Saunders Institute, London, UK

\subsection{6/bmjspcare-2017-hospice.197}

Background Up to $80 \%$ of people with advanced cancer experience cachexia and of these most have accompanying anorexia, characterised by poor appetite and weight loss. Cachexia has profound psychosocial impact on patients and families including negative emotions associated with reduced food intake and involuntary weight loss, and the social consequences of these symptoms. Weight loss can be unacknowledged by healthcare professionals (HCPs), even in settings where patients are weighed regularly. Little is known about the meaning of weight loss from the patient's perspective, and what support patients expect from HCPs.

Aim(s) - To describe the experience of weight loss in people with advanced cancer and explore what psychosocial support they believe HCPs could provide

- To make recommendations for clinical practice.

Methods Individual, semi-structured interviews with hospice patients diagnosed with advanced cancer and who had expressed distress around weight loss, analysed using thematic content analysis.

Results Nine patients took part. Participants described the emotional impact of weight loss as an indicator of overall deterioration or impending death. Concerns about weight loss were perceived to be unacknowledged by HCPs, and participants would welcome more opportunities to discuss their fears. Findings were categorised into four themes:

- The meaning of weight loss to the person

- A changed relationship with food

- The experience of being weighed as 'a tick box exercise'

- 'Listen to me' - a message for healthcare professionals. Conclusions HCPs should acknowledge weight loss in patients with advanced cancer to explore the impact and meaning. Increased awareness of the impact of cachexia and communication skills training may enable these difficult conversations to take place. With appropriate emotional and social support, patients and their families can identify and address what is important to them, which may reduce distress and improve quality of life.

\section{P-173 A ROLE FOR EMOTIONAL FREEDOM TECHNIQUE IN PALLIATIVE PATIENTS? THREE CASE REPORTS}

${ }^{1,2}$ Seamus Coyle. 'Willowbrook Hospice, Prescot, UK; ${ }^{2}$ MCPCIL, University of Liverpool

10.1136/bmjspcare-2017-hospice. 198

Background Emotional Freedom Technique (EFT) is a simple and common self-help technique, which is also known as 'Tapping'. It combines elements of exposure therapy, cognitive behavioural therapy and somatic stimulation. It is widespread in the public domain; Meta-analyses show that EFT is effective for anxiety, depression and Post Traumatic Stress Disorder (PTSD). There are no studies examining the effect of EFT for palliative patients.

Case Presentation This case report presents three cases of emotional distress in palliative patients.

Case Management Each patient was treated using EFT. Case Outcome Following treatment using EFT, all of the patients' emotional distress was decreased and within a very short time. Conclusions EFT is a very simple, effective and safe technique. EFT has the potential to be a powerful tool to improve the care of palliative patients who have distressing emotions. Future research on EFT and its effect on distressing emotions in palliative populations and their families is warranted.

\section{P-174 PERSONALISED CARE PLANNING}

Tracy Bell. Rotherham Hospice, Rotherham, UK

\subsection{6/bmjspcare-2017-hospice. 199}

Background It was identified that the hospice in- patient unit records were not as robust as they could be. Despite the patients receiving an excellent level of holistic care, this was not always evidenced clearly in the documentation.

Aim and Implementation To modify existing documentation to clearly evidence the excellent care being received by our inpatients and families, whilst being patient- centred and personalised. The time frame was set for compliance to be complete by the end of January 2017. Weekly audit to ensure the new documentation is fit for purpose and to identify any areas which need further development. Following the guidelines of the NMC code for nurses and midwives specifically sections: 10 - Keep clear and accurate records relevant to your practice ... 3 - Make sure that people's physical, social and psychological needs are assessed and responded to. 